Math. Model. Nat. Phenom.

Vol. 2, No. 2, 2007, pp. 106-125

\title{
Travelling Waves in Partially Degenerate Reaction-Diffusion Systems
}

\author{
B. Kazmierczak ${ }^{a 1}$ and V. Volpert ${ }^{b}$ \\ ${ }^{a}$ Institute of Fundamental Technological Research, PAS, Warsaw \\ ${ }^{b}$ Department of Mathematics, UMR 5208 CNRS, Université Lyon 1 \\ 69622 Villeurbanne, France
}

\begin{abstract}
We study the existence and some properties of travelling waves in partially degenerate reaction-diffusion systems. Such systems may for example describe intracellular calcium dynamics in the presence of immobile buffers. In order to prove the wave existence, we first consider the non degenerate case and then pass to the limit as some of the diffusion coefficient converge to zero. The passage to the limit is based on a priori estimates of solutions independent of the values of the diffusion coefficients. The wave uniqueness is also proved.
\end{abstract}

Key words: travelling waves, reaction-diffusion systems, calcium dynamics AMS subject classification: 34A34, 35K57

\section{Introduction}

In this paper we will analyze the problem of existence and properties of travelling wave solutions to the following system of reaction-diffusion equations:

$$
\begin{aligned}
& \frac{\partial u}{\partial t}=D \Delta u+f(u)+\sum_{i=1}^{n} G_{i}\left(u, v_{i}\right) \\
& \frac{\partial v_{i}}{\partial t}=D_{i} \Delta v_{i}-G_{i}\left(u, v_{i}\right), \quad i=1, \ldots, n \geq 1 .
\end{aligned}
$$

${ }^{1}$ Corresponding author. Email: bkazmier@ippt.gov.pl 
We want to show the existence of appropriate heteroclinic travelling wave solutions of the above system, when some of the diffusion coefficients converge to zero. Though such a problem may arise in many applications, our paper refers mainly to calcium dynamics in the presence of immobile buffers $([8],[10])$. We describe this phenomenon in a more detail below.

Assumption 1.1. Assume that:

1. The function $f(\cdot) \in C^{2}\left(\mathbb{R}^{1}\right)$ is of the bistable type, that is the equation $f(u)=0$ has exactly three solutions: $u_{1} \geq 0, u_{3}>u_{1}$ and $u_{2} \in\left(u_{1}, u_{3}\right)$. Moreover, $f^{\prime}\left(u_{1}\right)<0$, $f^{\prime}\left(u_{3}\right)<0$, and $f^{\prime}\left(u_{2}\right)>0$.

2. The functions $G_{i}(\cdot, \cdot), i=1, \ldots, n$ are of $C^{3}$ class. For each $k=1,2,3$, the equation

$$
G_{i}\left(u_{k}, v_{i}\right)=0, \quad i=1, \ldots, n,
$$

with respect to $v_{i}$ has a unique solution $v_{i}^{k}$, and $v_{i}^{1}<v_{i}^{2}<v_{i}^{3}$.

3. $G_{i, u}\left(u, v_{i}\right)<0$ and $G_{i, v_{i}}\left(u, v_{i}\right)>0$ for all $u \in\left[u_{1}, u_{3}\right], v_{i} \in\left[v_{i}^{1}, v_{i}^{3}\right], i=1, \ldots, n$.

Let us denote

$$
P_{k}=\left(u_{k}, v_{1}^{k}, \ldots, v_{n}^{k}\right), \quad k=1,2,3 .
$$

Thus component-wise

$$
P_{1}<P_{2}<P_{3}
$$

A simple example of a function satisfying the first condition of Assumption 1.1 is a cubic polynomial $f(u)=u(1-u)\left(u-u_{0}\right)$ with $u_{0} \in(0,1)$.

Let us take for example

$$
G_{i}\left(u, v_{i}\right)=k_{-}^{i} v_{i}-k_{+}^{i} u\left(b_{0}^{i}-v_{i}\right) .
$$

with $k_{-}^{i}, k_{+}^{i}>0$. Then system (1.1) describes the evolution of the concentration of free cytosolic calcium $(u)$ and the concentrations $v_{i}(i=1, \ldots, n)$ of the buffer proteins. Buffers are big proteins (e.g. parvalbumin, calsequestrin, calretinin or EGTA) which can bind a large amount of free calcium inside cells. The amount of free $\mathrm{Ca}^{2+}$ ions which can be bound to different kinds of buffers may reach $99 \%$. Most of the buffers are almost immobile, however the diffusion coefficient of some of them is not negligible and is in the range between onetenth to one-half of the diffusion coefficient for free $\mathrm{Ca}^{2+}[3]$. The action of buffers is to stabilize the concentration of the intracellular calcium in an appropriate range [3]. That is to say, in this case, $v_{i}=\left[\mathrm{Ca}^{2+} B_{i}\right]$ denotes the concentration of $i$-th kind of buffers, which have bound the calcium ions, and $b_{0}^{i}$ denotes the total concentration of the $i$-th buffer [8]. We have thus $b_{0}^{i}=\left[B_{i}\right]+\left[\mathrm{Ca}^{2+} B_{i}\right]$. For $G_{i}$ given by the expression (1.4) there are exactly three constant steady states of the considered system. They are of the form 


$$
P_{k}=\left(u_{k}, v_{1}^{k}, \ldots, v_{n}^{k}\right), \quad k=1,2,3
$$

where

$$
v_{j}^{k}=u_{k} \frac{k_{+}^{j} b_{0}^{j}}{\left(k_{-}^{j}+k_{+}^{j} u_{k}\right)} .
$$

It is easy to note that $P_{k}$ given by (1.5)-(1.6) satisfy inequality (1.3).

The results of the paper are twofold. First we prove the existence and some properties of the travelling wave solutions for the system of equations with positive diffusion coefficients $D, D_{1}, \ldots, D_{n}$. To do this we use the theory of travelling waves for parabolic systems contained in [11]. Then by applying appropriate limiting procedure we obtain the existence of waves for the system with non zero diffusion coefficients $D, D_{1}, \ldots, D_{M}, 0 \leq M \leq n-1$, and zero diffusion coefficients $D_{M+1}, \ldots, D_{n}$. The existence of travelling waves for the system with non-diffusing buffers, i.e for $M=0$ was proved recently in a straightforward way in the paper [10] and the asymptotic in time properties of solutions to this system were analyzed in [7]. It seems however that keeping non zero though arbitrarily small diffusion coefficients of buffers is more realistic in some situations. The method used in our paper allows us to consider the system with non diffusing as a limit of equations with small diffusing coefficients and provides some additional information about the behaviour of solutions to the considered family of systems. ¿From the point of view calcium dynamics in the presence of buffers, the generalization to the case $M \geq 1$ seems sensible due to the fact that some of the buffers may have non negligible diffusion coefficients.

Remark 1.1. Let us note that system (1.1) satisfies so called monotonicity conditions. To be more precise the derivative of the right-hand side of the equation for $u$ with respect to $v_{i}$, $i=1, \ldots, n$, is positive. Likewise, the derivative of the right-hand side of the equation for $v_{i}$ with respect to $u$ is positive whereas the derivative with respect to $v_{j}, j \neq i$, is identically zero. Thus it is possible to use the theory contained in [11].

In this paper we are interested in the travelling wave solutions to system (1.1) joining the constant steady states $P_{1}$ and $P_{3}$. To be more precise we are looking for solutions being functions of a scalar variable $\xi=x-q t$, that is

$$
u(x, t)=u(\xi), \quad v_{i}(x, t)=v_{i}(\xi)
$$

satisfying the following conditions:

$$
\begin{gathered}
\lim _{\xi \rightarrow-\infty}\left(u(\xi), v_{1}(\xi), \ldots, v_{n}(\xi)\right)=\left(u_{1}, v_{1}^{1}, \ldots, v_{n}^{1}\right)=P_{1} \\
\lim _{\xi \rightarrow \infty}\left(u(\xi), v_{1}(\xi), \ldots, v_{n}(\xi)\right)=\left(u_{3}, v_{1}^{3}, \ldots, v_{n}^{3}\right)=P_{3} \\
\lim _{|\xi| \rightarrow \infty}\left(u^{\prime}(\xi), v_{1}^{\prime}(\xi), \ldots, v_{n}^{\prime}(\xi)\right)=(0,0, \ldots, 0) .
\end{gathered}
$$

Assumption (1.7) changes Eqs (1.1) to the system 


$$
\begin{gathered}
D u^{\prime \prime}+q u^{\prime}+f(u)+\sum_{i=1}^{n} G_{i}\left(u, v_{i}\right)=0, \\
D_{i} v_{i}^{\prime \prime}+q v_{i}^{\prime}-G_{i}\left(u, v_{i}\right)=0, \quad i=1, \ldots, n,
\end{gathered}
$$

where' denotes differentiation with respect to the variable $\xi$.

As we mentioned above our main aim was to prove the existence of heteroclinic solutions for a partially degenerated version of the system (1.9)-(1.10), that is the system

$$
\begin{gathered}
D u^{\prime \prime}+q u^{\prime}+f(u)+\sum_{i=1}^{n} G_{i}\left(u, v_{i}\right)=0, \\
D_{i} v_{i}^{\prime \prime}+q v_{i}^{\prime}-G_{i}\left(u, v_{i}\right)=0, \quad i=1, \ldots, M, \\
q v_{i}^{\prime}-G_{i}\left(u, v_{i}\right)=0, \quad i=M+1, \ldots, n .
\end{gathered}
$$

The method of the proof is based on the passage to the limit in the family of solutions when the diffusion coefficients $D_{i}>0, i=M+1, \ldots, n$ converge to zero. The existence of heteroclinic solutions to system (1.9)-(1.10) with $D_{i}>0$ is guaranteed by the theory contained in [11].

In the proof we will refer to the properties of the subsystem describing the evolution of $u$ and $v_{i}, i \in\{1, \ldots, M\}$ that is to say of those $v_{i}$ for which the diffusion coefficients stay positive in the limiting process. This system has the following form:

$$
\begin{gathered}
D u^{\prime \prime}+q u^{\prime}+f(u)+\sum_{i=1}^{M} G_{i}\left(u, v_{i}\right)=0, \\
D_{i} v_{i}^{\prime \prime}+q v_{i}^{\prime}-G_{i}\left(u, v_{i}\right)=0, \quad i=1, \ldots, M .
\end{gathered}
$$

It is easy to note that the constant steady states for system (1.14)-(1.15), which will be denoted by $\mathcal{P}_{1}, \mathcal{P}_{2}, \mathcal{P}_{3}$, satisfy the relations (1.3) with $j \in\{1, \ldots, M\}$. Similarly to the heteroclinic solutions joining the states $P_{1}$ and $P_{3}$ for system (1.9)-(1.10) one can consider the heteroclinic solutions of system (1.14)-(1.15) joining its constant states $\mathcal{P}_{1}$ and $\mathcal{P}_{3}$. According to Theorem 2.1 p.15 in [11] there exists a unique (up to a translation in $\xi$ ) heteroclinic pair $\left(q_{r}, U_{r}\right)$ for system (1.9)-(1.10) satisfying conditions (1.8) with $P_{1}, P_{3}$ replaced by $\mathcal{P}_{1}, \mathcal{P}_{3}$ and $n$ replaced by $M$.

\section{Properties of the constant states}

To begin with we will analyze the properties of the matrices obtained by linearization of the source terms at the right-hand sides of the system (1.1) at the points $P_{k}, k=1,2,3$. To be more precise, we are interested in the properties of their eigenvalues and eigenvectors. 
Consider the following $(n+1) \times(n+1)$ matrix:

$$
K=\left[\begin{array}{cccc}
a-\sum_{i=1}^{n} a_{i} & b_{1} & \ldots & b_{n} \\
a_{1} & -b_{1} & \ldots & 0 \\
\cdot . & . & \ldots & . . \\
a_{n} & 0 & \ldots & -b_{n}
\end{array}\right]
$$

where

$$
a_{i}>0, \quad b_{i}>0, \quad i=1, \ldots, n .
$$

One can easily check that for $u=u_{k}+\delta u, v_{j}=v_{j}^{k}+\delta v_{j}$ the first order Taylor expansion of the source terms of system (1.1) has the form

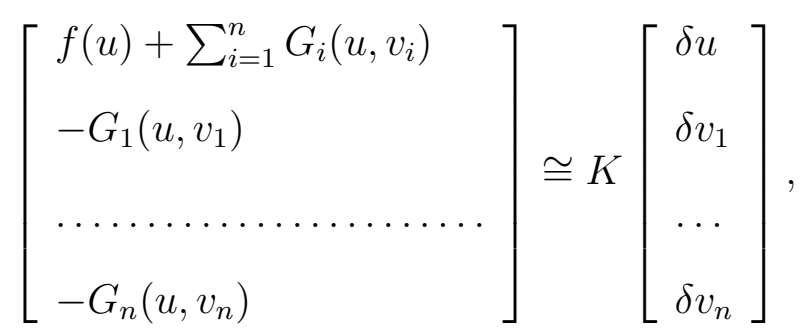

where $K$ has the form defined by (2.1) with

$$
a=f_{, u}\left(u_{k}\right), a_{i}=-G_{i, u}\left(u_{k}, v_{i}^{k}\right), \quad b_{i}=G_{i, v_{i}}\left(u_{k}, v_{i}^{k}\right) .
$$

Obviously, for $a_{i}, b_{i}$ given by the above expressions the relations (2.2) are satisfied.

Below $N \geq 1$ will denote a natural number.

Lemma 2.1. (see Theorem 5 in [4], p.350) Let $C$ be a real $N \times N$-matrix with nonnegative off-diagonal entries $c_{i k} \geq 0, i \neq k$. Then all the eigenvalues of $C$ have negative real parts if and only if the following inequalities are satisfied

$$
J_{1}=c_{11}<0, \quad J_{2}=\left|\begin{array}{cc}
c_{11} & c_{12} \\
c_{21} & c_{22}
\end{array}\right|>0, \ldots \ldots, J_{N}=(-1)^{N}\left|\begin{array}{cccc}
c_{11} & c_{12} & \ldots & c_{1 N} \\
c_{21} & c_{22} & \ldots & c_{2 N} \\
. . & . . & \ldots & . . \\
c_{N 1} & c_{N 2} & \ldots & c_{N N}
\end{array}\right|>0
$$

Lemma 2.2. (see [4], Theorem 6, p.350) Let $C$ be an $N \times N$ matrix with nonnegative elements. Then its maximal eigenvalue is a strictly increasing function of any of its entries.

This maximal eigenvalue is characterized in the following lemma. 
Lemma 2.3. (see [4], Theorem 3, p.344, Theorem 2 p.334) Let $C$ be an $N \times N$ matrix with nonnegative entries. Then $C$ has a real nonnegative eigenvalue $\mu_{P F}(C)$ such that an associated eigenvector has nonnegative components and every other eigenvalue of $C$ has its modulus less than $\mu_{P F}(C)$. In particular every other eigenvalue of $C$ has its real part less than $\mu_{P F}$. Moreover, if $C$ is irreducible, then the associated eigenvector has positive components.

We note that a matrix with positive elements is irreducible. Therefore its principal eigenvalue is positive up to a constant factor. Lemma 2.3 can be generalized for the matrices with non-negative off-diagonal elements.

Lemma 2.4. Let $C$ be a real $N \times N$ with nonnegative off-diagonal entries. Then $C$ has a real eigenvalue $\mu_{P F}(C)$ such that an associated eigenvector has nonnegative components and every other eigenvalue of $C$ has its real part less than $\mu_{P F}(C)$.

Proof. Let us consider the matrix $C_{m}=C+m I$, where $I$ is the unit $N \times N$ matrix and $m \in \mathbb{R}^{1}$. Every eigenvalue $\mu$ of $C$ determines an eigenvalue $\mu_{m}$ of the matrix $C_{m}$ by the relation

$$
\mu_{m}=m+\mu \text {. }
$$

If $m$ is taken sufficiently large then the matrix $C_{m}$ has nonnegative entries. According to Lemma 2.3 there exists a maximal real and positive eigenvalue $\mu_{P F}(C+m I)$ of the matrix $C+m I$ and the corresponding eigenvector $\mathbf{E}$ has nonnegative entries. Consequently the matrix $C$ possesses a maximal real eigenvalue $\mu_{P F}(C)$ equal to $\mu_{P F}(C+m I)-m$ with the corresponding eigenvector $\mathbf{E}$, as $C \mathbf{E}=\left(C_{m}-m I\right) \mathbf{E}=\left(\mu_{P F}(C+m I)-m\right) \mathbf{E}=\mu_{P F}(C) \mathbf{E}$. The lemma is proved.

Definition 1. Let $C$ be a real $N \times N, N \geq 2$, matrix with nonnegative off-diagonal entries. Then the eigenvalue $\mu_{P F}(C)$ is called the Perron-Frobenius or principal eigenvalue of $C$ and an associated eigenvector (with nonnegative components) a Perron-Frobenius or principal eigenvector of $C$.

Lemma 2.5. Let $C$ be a real $N \times N$ matrix with nonnegative off-diagonal elements. Then its principal eigenvalue is a strictly increasing function of any of its entries.

Proof. The proof follows by repeating the proof of Lemma 2.4 and using Lemma 2.2.

According to inequalities (2.2) the matrix $K$ has positive off-diagonal entries. Using Lemma 2.1 we can prove that for $a<0$ its maximal eigenvalue $\mu_{P F}(K)$ is negative.

Lemma 2.6. Let $a<0$. Then the eigenvalues $\mu$ of the matrix $K$ are contained in the left half-plane $\operatorname{Re}(\mu)<0$.

Proof. Let us note that $K_{11}<0$. Consider the matrix $K^{k}=K_{i j}, i, j=1, \ldots, k, k=$ $2, \ldots, n+1$. Let us compute the determinant of the matrix $K^{k}$. To this end, let us add the last $k-1$ rows of the matrix $K^{k}$ to its first row. Expanding the determinant of this matrix 
with respect to the first row, we immediately obtain det $K^{k}=\left(a-\sum_{j=k}^{n} a_{j}\right)(-1)^{k-1} b_{1} \ldots b_{k-1}$. Its sign is equal to $(-1)^{k}$. Also $\operatorname{det} K^{n+1}=\operatorname{det} K=(a)(-1)^{n} b_{1} \ldots b_{n}$. Thus the sequence of inequalities from Lemma 2.1 is satisfied. The lemma is proved.

The statement converse to Lemma 2.6 is also true. Namely, the following lemma holds.

Lemma 2.7. Let $a>0$. Then at least one of the eigenvalues $\mu$ of the matrix $K$ is contained in the half-plane $\operatorname{Re}(\mu)>0$.

Proof. Let us consider the matrix $K$ with $a=0$. Then (by adding to the first row all the other rows) we note that $\operatorname{det}(K)=0$. Now, the claim of the lemma follows from Lemma 2.5 .

Now, we will analyze the irreducibility of the matrix $K$.

Lemma 2.8. Independently of the value of a the matrix $K$ is irreducible.

Proof. Let us remind that an $(n+1) \times(n+1)$ matrix $C$ is called reducible if the set $\{1, \ldots, n+1\}$ can be divided into two disjoint subsets $I$ and $J$, that is to say $\{1, \ldots, n+1\}=$ $I \cup J, I \cap J=\emptyset$ such that $c_{i j}=0$ for all $i \in I, j \in J$. The matrix is called irreducible, if it is not reducible.

Suppose to the contrary that $K$ is reducible for some value of $a$. It follows from the definition of reducibility that $i \in I$ implies $i \notin J$ and vice versa $j \in J$ implies $j \notin I$. As $k_{1 j} \neq 0$ for $j \neq 1$ we note that $1 \notin I$. Hence $1 \in J$. Consequently there must exist $1 \neq i \in I$ such that $k_{i 1}=0$. But $k_{i 1}$ can be equal to zero only for $i=1$. We thus arrive at contradiction which proves the irreducibility of $K$ independently of the value of $a$.

Using Lemmas 2.3, 2.8 and the method used in the proof of Lemma 2.4 we can easily note the validity of the following statement.

Lemma 2.9. Let $K$ be defined by conditions (2.1)-(2.2). Then for any a its principal eigenvector (corresponding to the principal eigenvalue) is positive up to a constant factor.

\section{Existence theorem for the non degenerate system}

The following theorem holds.

Theorem 3.1. (Theorem 2.1, p.15 in [11]) Let us consider the system

$$
\frac{\partial U}{\partial t}=A \Delta U+F(U)
$$

where $U=\left(U_{1}, \ldots, U_{N}\right)$ is a vector-valued function, $A$ is a diagonal positive definite matrix and $C^{1} \ni F(\cdot): \mathbb{R}^{N} \rightarrow \mathbb{R}^{N}$. Let system (3.1) be monotone, that is 


$$
\frac{\partial F_{i}}{\partial U_{j}} \geq 0, \quad i, j=1, \ldots, N, i \neq j .
$$

Further, let the function $F(U)$ vanish in a finite number of points $w_{-}, w_{+}$and $\mathbf{U}_{k},(k=$ $1, \ldots, m)$ with $w_{-}<\mathbf{U}_{k}<w_{+}$. Let us assume that all the eigenvalues of the matrices $\partial F\left(w_{-}\right)$and $\partial F\left(w_{+}\right)$lie in the left half-plane, and that the matrices $F\left(\mathbf{U}_{k}\right),(k=1, \ldots, m)$ are irreducible and have at least one eigenvalue in the right half-plane. Then there exists a unique monotone travelling wave, that is a constant $q$ and a twice continuously differentiable monotone vector-valued function $U(\xi), \xi=x-q t$, satisfying the system

$$
A U^{\prime \prime}+q U^{\prime}+F(U)=0,
$$

such that $U_{j}^{\prime}(\xi)>0$ for all $j=1, \ldots, N, \xi \in \mathbb{R}^{1}$, and

$$
\lim _{\xi \rightarrow \pm \infty} U(\xi)=w_{ \pm}, \quad \lim _{\xi \rightarrow \pm \infty} U^{\prime}(\xi)=0
$$

By means of the results of Section 2 and Theorem 3.1 one can easily check the validity of the existence theorem for the travelling wave solutions to system (1.1) with positive diffusion coefficients.

Theorem 3.2. Let Assumption 1.1 be satisfied. Let $D, D_{1}, \ldots, D_{n}$ be positive. Then there exists a unique monotone heteroclinic solution to system (1.9)-(1.10) satisfying conditions (1.8).

\section{A priori estimates of the solutions}

Now using the special structure of the considered system we will estimate the derivatives of the heteroclinic solutions to system (1.9)-(1.10) together with the speed $q$. Our aim is mainly to examine the properties of the heteroclinic solutions as the coefficients $D_{i}, i=M+1, \ldots, n$, tend to zero. The estimates obtained in this section will be used to prove the existence of a solution to the system with $D_{i}=0, i=M+1, \ldots, n$.

Lemma 4.1. For any $C^{2}$ monotone heteroclinic solution $\left(u, v_{1}, \ldots, v_{n}\right)(\cdot)$ of system (1.9)(1.10) we have the estimates

$$
\begin{gathered}
\|u\|_{C^{3}\left(\mathbb{R}^{1}\right)}<C_{u}, \quad\left\|v_{i}\right\|_{C^{3}\left(\mathbb{R}^{1}\right)}<C_{v}, \quad i=1, \ldots, M, \\
\left\|v_{i}\right\|_{C^{2}\left(\mathbb{R}^{1}\right)}<C_{v}, \quad i=M+1, \ldots, n .
\end{gathered}
$$

The constants $C_{u}$ and $C_{v}$ can be chosen independent of $q$. 
Proof. First let us estimate the first derivative of the function $u(\cdot)$. Let us suppose that the supremum of $u^{\prime}$ is attained for some $\xi_{0} \in \mathbb{R}^{1}$. As $\lim _{\xi \rightarrow \pm \infty} u^{\prime}(\xi)=0$, hence we must have $u^{\prime \prime}\left(\xi_{0}\right)=0$ and $q=-G\left(u\left(\xi_{0}\right), v_{1}\left(\xi_{0}\right), \ldots, v_{n}\left(\xi_{0}\right)\right)\left(u^{\prime}\left(\xi_{0}\right)^{-1}\right)$ where we have denoted

$$
G\left(u, v_{1}, \ldots, v_{n}\right)=f(u)+\sum_{i=1}^{n} G_{i}\left(u, v_{i}\right) .
$$

Multiplying Eq.(1.9) by $u^{\prime}(\xi)$ and integrating on $\left(-\infty, \xi_{0}\right)$ we obtain the equality

$$
\begin{aligned}
\frac{1}{2} D u^{\prime 2}\left(\xi_{0}\right) & =G\left(u\left(\xi_{0}\right), v_{1}\left(\xi_{0}\right), \ldots, v_{n}\left(\xi_{0}\right)\right) \int_{-\infty}^{\xi_{0}} \frac{u^{\prime}(\xi)}{u^{\prime}\left(\xi_{0}\right)} u^{\prime}(\xi) d \xi \\
& -\int_{-\infty}^{\xi_{0}} G\left(u(\xi), v_{1}(\xi), \ldots, v_{n}(\xi)\right) u^{\prime}(\xi) d \xi .
\end{aligned}
$$

Hence, due to the monotonicity of the functions $u, v_{1}, \ldots, v_{n}$, and the continuity of the function $G$, the right-hand side of this equation can be estimated from above by the expression

$$
C \int_{-\infty}^{\xi_{0}} u^{\prime}(\xi) d \xi \leq C u\left(\xi_{0}\right)
$$

where $C$ is a constant independent of the value of $\xi_{0}$. As $D>0$ we obtain from here the global boundedness of the $C^{1}$ norm of the function $u(\cdot)$ independently of the value of $q$. In the same way we can prove the estimate for $v_{i}^{\prime}(\cdot), i=1, \ldots, M$. Next, note that for all $\xi \in \mathbb{R}^{1}$ we have $\left|q u^{\prime}(\xi)\right| \leq\left|q u^{\prime}\left(\xi_{0}\right)\right|$ (where $\xi_{0}$ is the point of supremum of $u^{\prime}$ ). Likewise, for $i=1, \ldots, M$, $\left|q v_{i}^{\prime}(\xi)\right| \leq\left|q v_{i}^{\prime}\left(\xi_{0}\right)\right|$ for some $\xi_{0}$. The quantities on the right-hand sides of these inequalities are determined only by the properties of the functions $G$ and $G_{i}$ respectively. Thus using the equations for $u$ and $v_{i}, i=1, \ldots, M$, we can estimate also the second derivatives of these functions. As a result we have proved that

$$
\|u\|_{C^{2}\left(\mathbb{R}^{1}\right)}<C_{2 u}, \quad\left\|v_{i}\right\|_{C^{2}\left(\mathbb{R}^{1}\right)}<C_{2 v}, \quad i=1, \ldots, M .
$$

Using the results obtained above, we will now prove that the $C^{1}$ norms of the functions $v_{k}$, $k=M+1, \ldots, n$, are also bounded without imposing any lower bounds for the coefficients $D_{k}>0$. Suppose that $v_{k}^{\prime}(\cdot)$ attains its global maximum at $\xi=\xi_{0}$. (Then $\xi_{0}$ must be finite as $v_{k}^{\prime}(\xi) \rightarrow 0$ for $\xi \rightarrow \pm \infty$.) Differentiating the $k$-th $(k=M+1, \ldots, n)$ equation of system (1.10) with respect to $\xi$ we obtain the following equation

$$
\begin{aligned}
& 0=D_{k} v_{k}^{\prime \prime \prime}\left(\xi_{0}\right)-G_{k, v_{k}}\left(u\left(\xi_{0}\right), v_{k}\left(\xi_{0}\right)\right) v_{k}^{\prime}\left(\xi_{0}\right) \\
& -G_{k, u}\left(u\left(\xi_{0}\right), v_{k}\left(\xi_{0}\right)\right) u^{\prime}\left(\xi_{0}\right) .
\end{aligned}
$$

As $\xi_{0}$ is the point of the global maximum of $v_{k}^{\prime}$ we must have $v_{k}^{\prime \prime \prime}\left(\xi_{0}\right) \leq 0$. Now, independently of $\xi_{0}$,

$$
G_{k, v_{k}}\left(u\left(\xi_{0}\right), v_{k}\left(\xi_{0}\right)\right) \geq B_{k}>0
$$


(see point 3. of Assumption 1.1) so the sum of the first two terms at the right hand side can be annihilated only by the third term as $-G_{k, u}\left(u\left(\xi_{0}\right), v_{k}\left(\xi_{0}\right)\right)>0$. However, the last quantity is bounded for any $P_{1} \leq(u, v) \leq P_{3}$. Thus also $v_{k}^{\prime}(\cdot)$ must be bounded in its $C^{1}$ norm for all $k \in\{M+1, \ldots, n\}$.

Finally, let us estimate the behavior of the second derivatives of the functions $v_{k}, k=$ $M+1, \ldots, n$, independently of the values of $D_{k}>0$. These estimates are also based on inequalities (4.4). Namely, differentiating twice the equation for $v_{k}, k=M+1, \ldots, n$, (see Remark ref1) we obtain for $\xi=\xi_{0}$

$$
\begin{aligned}
& 0=D_{k} v_{k}^{\prime \prime \prime \prime}\left(\xi_{0}\right)+q v_{k}^{\prime \prime \prime}\left(\xi_{0}\right)-G_{k, v_{k}}\left(u\left(\xi_{0}\right), v_{k}\left(\xi_{0}\right)\right) v_{k}^{\prime \prime}\left(\xi_{0}\right)- \\
& G_{k, v_{k} v_{k}}\left(u\left(\xi_{0}\right), v_{k}\left(\xi_{0}\right)\right) v_{k}^{\prime}\left(\xi_{0}\right) v_{k}^{\prime}\left(\xi_{0}\right)-G_{k, u}\left(u\left(\xi_{0}\right), v_{k}\left(\xi_{0}\right)\right) u^{\prime \prime}\left(\xi_{0}\right)- \\
& 2 G_{k, u v_{k}}\left(u\left(\xi_{0}\right), v_{k}\left(\xi_{0}\right)\right) u^{\prime}\left(\xi_{0}\right) v_{k}^{\prime}\left(\xi_{0}\right)-G_{k, u u}\left(u\left(\xi_{0}\right), v_{k}\left(\xi_{0}\right)\right) u^{\prime}\left(\xi_{0}\right) u^{\prime}\left(\xi_{0}\right) .
\end{aligned}
$$

(In fact $G_{k, u u} \equiv 0$, but it is not essential.) Suppose that $v_{k}^{\prime \prime}$ attains its global maximum (minimum) at $\xi=\xi_{0}$. Then $v_{k}^{\prime \prime \prime}\left(\xi_{0}\right)=0$ and $v_{k}^{\prime \prime \prime \prime}\left(\xi_{0}\right) \leq 0\left(v_{k}^{\prime \prime \prime \prime}\left(\xi_{0}\right) \geq 0\right)$. Thus using the inequality (4.4) we infer that $\left|v_{k}^{\prime \prime}\left(\xi_{0}\right)\right|$ can be estimated by $|u|_{C^{2}\left(\mathbb{R}^{1}\right)}$ and $|v|_{C^{1}\left(\mathbb{R}^{1}\right)}$.

Now, differentiating the equations for $u, v_{1}, \ldots, v_{M}$ and using the arguments leading to the estimates of the second derivatives of these functions, we obtain the estimates of their third derivatives. We have thus completed the estimates (4.1). The lemma is proved.

The estimates of the derivatives of the monotone solutions allow us to estimate the absolute value of the wave speed $q$. To simplify the notation we will use the notation

$$
\begin{aligned}
& U:=\left(U_{1}, U_{2}, \ldots, U_{n+1}\right):=\left(u, v_{1}, \ldots, v_{n}\right), \quad F_{1}(U):=G\left(u, v_{1}, \ldots, v_{n}\right), \\
& F_{j}(U):=-G_{j-1}\left(u, v_{j-1}\right), \quad j=2, \ldots, n+1, \quad F(U):=\left(F_{1}(U), F_{2}(U), \ldots, F_{n+1}(U)\right),
\end{aligned}
$$

with $G$ defined in (4.2).

Lemma 4.2. Let $F$ be defined as above. Let $N\left(P_{1}\right)$ and $N\left(P_{3}\right)$ denote the eigenvectors corresponding to the Perron-Frobenius eigenvalue of $\partial F\left(P_{1}\right)$ and $\partial F(P 3)$ respectively. Then there exist $r>0$ and $\vartheta>0$ such that for each $i \in\{1, \ldots, n+1\}$

$$
\begin{aligned}
& \operatorname{dist}\left(U, W_{0 i}\right)<\vartheta(r) \Longrightarrow F_{i}(U)<0, \\
& \operatorname{dist}\left(U, W_{1 i}\right)<\vartheta(r) \Longrightarrow F_{i}(U)>0,
\end{aligned}
$$

where

$$
\begin{aligned}
& W_{0 i}=\left\{U: P_{1} \leq U \leq P_{1}+r N\left(P_{1}\right), U_{i}=\left[P_{1}+r N\left(P_{1}\right)\right]_{i}\right\}, \\
& W_{1 i}=\left\{U: P_{3} \geq U \geq P_{3}-r N\left(P_{3}\right), U_{i}=\left[P_{3}-r N\left(P_{3}\right)\right]_{i}\right\} .
\end{aligned}
$$


Proof. Let $\mu_{P F}$ denote the Perron-Frobenius eigenvalue of $\partial F\left(P_{1}\right)$. Then $r$ may be taken so small that $F\left(P_{1}+r N\left(P_{1}\right)\right)=r \partial F N\left(P_{1}\right)+o(r)<\frac{1}{2} r \mu_{P F} N\left(P_{1}\right)$. By means of conditions (2.1),(2.2) we conclude that $F_{i}(U)<0$ for $U \in W_{0 i}$. Consequently, there exists $\vartheta=\vartheta(r)>0$ such that the first of the above relations is satisfied. In the same way we prove the second relation.

Lemma 4.3. Let $F$ and $r$ be the same as in Lemma 4.2. Then for any point $U \in W_{0}=$ $\left\{U: P_{1} \leq U \leq P_{1}+r N\left(P_{1}\right), U \neq P_{1}\right\}$ there exists $i \in\{1, \ldots, n+1\}$ such that $F_{i}(U)<0$. Likewise, for any point $U \in W_{1}=\left\{U: P_{3} \geq U \geq P_{3}-r N\left(P_{3}\right), U \neq P_{3}\right\}$ there exists $i \in\{1, \ldots, n+1\}$ such that $F_{i}(U)>0$.

Proof. Let us take an arbitrary point $U=\left(U_{1}, \ldots, U_{n+1}\right) \in W_{0}$. Let

$$
\widetilde{r}=\max _{j}\left(U_{j}-P_{1 j}\right)\left(r N_{j}\left(P_{1}\right)\right)^{-1}=\left(U_{k}-P_{1 k}\right)\left(r N_{k}\left(P_{1}\right)\right)^{-1}
$$

for some $k \in\{1, \ldots, n+1\}$. (Let us remind that the components of the Perron-Frobenius eigenvectors are strictly positive according to Lemma 2.9.) As $\widetilde{r} \leq r$ then it follows from the proof of Lemma 4.2 that it holds with $r$ replaced by $\widetilde{r}$ and $\vartheta(r)$ replaced by $\vartheta(\widetilde{r})$. Consequently, $F_{k}(U)<0$ for $U \in \widetilde{W}_{0 k}=\left\{U: P_{1} \leq U \leq P_{1}+\widetilde{r} N\left(P_{1}\right), U_{k}=P_{1 k}+\widetilde{r} N_{k}\left(P_{1}\right)\right\}$. In the same way we consider the parallelepiped $W_{1}$. The lemma is proved.

The proofs of Lemmas 4.2 and 4.3 are based on considerations in [1], [11]. As a corollary to Lemma 4.3 we have the following lemma.

Lemma 4.4. Let $F$ be the same as in Lemma 4.2. Then there does not exist a point $U, 0<|U|<\widetilde{\delta}, \widetilde{\delta}$ sufficiently small, such that $F_{i}(U) \geq 0$ for all $i \in\{1, \ldots, n+1\}$. Likewise there does not exist a point $U, 0<\left|P_{3}-U\right|<\widetilde{\delta}$, $\widetilde{\delta}$ sufficiently small, such that $F_{i}(U) \leq 0$ for all $i \in\{1, \ldots, n+1\}$.

Proof. It suffices to take $\widetilde{\delta}<r$ and apply Lemma 4.3 .

We are able now to obtain a priori estimates for $q$.

Lemma 4.5. If $(q, U(\cdot))$ is a strictly monotone heteroclinic pair for system (1.9)-(1.10), then $|q|<Q$, where $Q$ is independent of $U$.

Proof. Since $U(\xi) \rightarrow P_{1}$ monotonically as $\xi \rightarrow-\infty$, then there must exist an index $i$ and $\xi=\xi_{0}$ such that $U(\xi)$ enters the region $P_{1} \leq U \leq P+r N\left(P_{1}\right)$ through the (closed) set $W_{0 i}$, i.e. $U\left(\xi_{0}\right) \in W_{0 i}$ (see Lemma 4.2). Let us take $\xi_{1}<\xi_{0}$ such that $U_{i}\left(\xi_{0}\right)-U_{i}\left(\xi_{1}\right)=\frac{\vartheta}{2}$. Integrating the $i$-th equation of system (1.9)-(1.10) we obtain

$$
R_{1}+q \frac{\vartheta}{2}+\int_{\xi_{1}}^{\xi_{0}} F_{i}(U(s)) d s=0,
$$

where $\left|R_{1}\right| \in\left(0, \max \left\{C_{u}, C_{v}\right\}\right)$ according to Lemma 4.1 and $F_{i}(U(s))<0$ for $s \in\left(\xi_{1}, \xi_{0}\right)$ according to Lemma 4.4. If $q \leq 0$ then $q \geq-2 R_{1} \vartheta^{-1}$. If $q>0$, then the analysis of the behavior of the heteroclinic trajectory near the $P_{3}$ allows us to obtain the upper bound for $q$. 


\section{Existence of a solution to the degenerate system}

In this section we will prove the existence of a heteroclinic solution of system (1.9)-(1.10) with $D_{i}=0$ for $i=M+1, \ldots, n$. The proof will be carried out by passing to the limit $D_{i} \rightarrow 0$ in the family of solutions for the non degenerate system (with $D_{i}>0$ ).

For $D_{i}=0, i=M+1, \ldots, n$, and for $q \neq 0$, system (1.9)-(1.10) can be written as a first order system of ODEs of the form

$$
\begin{aligned}
u^{\prime} & =z \\
v_{i}^{\prime} & =z_{i}, \quad i=1, \ldots, M, \\
z^{\prime} & =\frac{1}{D}\left[-q z-f(u)-\sum_{k=1}^{n} G_{k}\left(u, v_{k}\right)\right], \\
z_{i}^{\prime} & =\frac{1}{D_{i}}\left[-q z_{i}+G_{i}\left(u, v_{i}\right)\right], \quad i=1, \ldots, M, \\
v_{i}^{\prime} & =\frac{1}{q} G_{i}\left(u, v_{i}\right), \quad i=M+1, \ldots, n .
\end{aligned}
$$

We will also consider the system linearized around the point

$$
(u_{2}, v_{1}^{2}, \ldots, v_{M}^{2}, 0, \underbrace{0, \ldots, 0}_{M \text { times }}, v_{M+1}^{2}, \ldots, v_{n}^{2})
$$

(which corresponds to $P_{2}$ ):

$$
Z^{\prime}=L Z
$$

where $Z=\left(h_{u}, h_{* 1}, \ldots, h_{* M}, h_{z}, h_{1}, \ldots, h_{n}\right)^{T}$,

$$
L=\left(\begin{array}{ccccccccccc}
0 & 0 & \ldots & 0 & 1 & 0 & \ldots & 0 & 0 & \ldots & 0 \\
0 & 0 & \ldots & 0 & 0 & 1 & \ldots & 0 & 0 & \ldots & 0 \\
\ldots & \ldots & \ldots & \ldots & \ldots & \ldots & \ldots & \ldots & \ldots & \ldots & \ldots \\
0 & 0 & \ldots & 0 & 0 & 0 & \ldots & 1 & 0 & \ldots & 0 \\
\frac{1}{D}\left[-a+\sum_{i=1}^{n} a_{i}\right] & -\frac{1}{D} b_{1} & \ldots & -\frac{1}{D} b_{M} & -\frac{1}{D} q & 0 & \ldots & 0 & -\frac{1}{D} b_{M+1} & \ldots & -\frac{1}{D} b_{n} \\
-\frac{1}{D_{1}} a_{1} & \frac{1}{D_{1}} b_{1} & \ldots & 0 & 0 & -\frac{1}{D_{1}} q & \ldots & 0 & 0 & \ldots & 0 \\
\ldots & \ldots & \ldots & \ldots & \ldots & \ldots & \ldots & \ldots & \ldots & \ldots & \ldots \\
-\frac{1}{D_{M}} a_{M} & 0 & \ldots & \frac{1}{D_{M}} b_{M} & 0 & 0 & \ldots & -\frac{1}{D_{M}} q & 0 & \ldots & 0 \\
-\frac{1}{q} a_{M+1} & 0 & \ldots & 0 & 0 & 0 & \ldots & 0 & \frac{1}{q} b_{M+1} & \ldots & 0 \\
\ldots & \ldots & \ldots & \ldots & \ldots & \ldots & \ldots & \ldots & \ldots & \ldots & \ldots \\
-\frac{1}{q} a_{n} & 0 & \ldots & 0 & 0 & 0 & \ldots & 0 & 0 & \ldots & \frac{1}{q} b_{n}
\end{array}\right),
$$

with $a, a_{i}$ and $b_{i}$ satisfying (2.2) and (2.4) for $k=2$.

First we will prove that while decreasing the coefficients $D_{i}, i=M+1, \ldots, n$, to zero the heteroclinic solution joining $P_{1}$ with $P_{3}$ cannot split into two waves joining in turn the points 
$P_{1}$ with $P_{2}$ and $P_{2}$ with $P_{3}$. (For the details see [5], p.478.) Suppose to the contrary that the above splitting into the two heteroclinics is possible. These waves would be a limit of heteroclinics with positive first derivatives. Then, for a fixed $q \neq 0$ there would exist simultaneously:

1. nonnegative eigenvalue $\lambda_{+}$of the matrix (5.3) with $a>0$ and a corresponding eigenvector $N_{2+}=\left(h_{u}, h_{* 1}, \ldots, h_{* M}, h_{z}, h_{1}, \ldots, h_{n}\right)^{T}$ with nonnegative components

2. non positive eigenvalue $\lambda_{-}$of the matrix (5.3) with $a>0$ and a corresponding eigenvector $N_{2-}=\left(h_{u}, h_{* 1}, \ldots, h_{* M}, h_{z}, h_{1}, \ldots, h_{n}\right)^{T}$ with $h_{u} \geq 0, h_{* k} \geq 0, h_{z} \leq 0, h_{k} \leq 0$ for $k=1, \ldots, M$ and and $h_{k} \geq 0$ for $k=M+1, \ldots, n$.

Their existence follows from the fact the first heteroclinic trajectory starts from $P_{2}$ into the set $\left\{\left(U: U-P_{2} \geq 0\right.\right.$ componentwise $\}$ as $\xi$ increases from $(-\infty)$, whereas the second trajectory achieves $P_{2}$ from the set $\left\{\left(U: U-P_{2} \leq 0\right.\right.$ componentwise $\}$ as $\xi$ tends to $(\infty)$.

The following lemma holds.

Lemma 5.1. For a fixed $q \neq 0$ the properties 1 and 2 cannot be fulfilled simultaneously.

Lemma 5.2. Assume that $a<0$ and $a_{k}, b_{k}>0$. Then no eigenvalue of the matrix (5.3) can have its real part equal to zero.

Proof. Let $\hat{D}:=\operatorname{diag}\left(D, D_{1}, \ldots, D_{M}, 0, \ldots, 0\right)$. Suppose to the contrary that there exists an eigenvector $\widetilde{N} \in \mathbb{R}^{n+M+2}$ of matrix (5.3) corresponding to a purely imaginary pair of eigenvalues $\lambda= \pm \mathbf{i} \kappa$. Now, let $N:=\left(h_{u}, h_{* 1}, \ldots, h_{* M}, h_{M+1}, \ldots, h_{n}\right)^{T}$ (in the notation of Eq.(5.2)), where $h_{u}, h_{* 1}, \ldots, h_{* M}, h_{M+1}, \ldots, h_{n}$ are the components of the eigenvector $\tilde{N}$. Then it is easy to see that the eigenvalue $\mathbf{i} \kappa$ and $N$ would satisfy the relation:

$$
\left(-\hat{D} \kappa^{2}+\mathbf{i} q \kappa+\partial F\right) N=0
$$

which can be written as

$$
\left(-\hat{D} \kappa^{2}+\partial F\right) N=-\mathbf{i} q \kappa N
$$

This means that the matrix $\left(-\hat{D} \kappa^{2}+\partial F\right)$ has a purely imaginary eigenvalue and the corresponding eigenvector $N$. But this impossible due to Lemmas 2.5 and 2.6.

When $D>0, D_{i}>0, i=1, \ldots, n$, every component of the heteroclinic solutions $U(\xi)$ provided by Theorem 3.2 are monotonically increasing. Consequently the solutions to system (5.1), if they exist, obtained by passing to the limit $D_{i} \rightarrow 0$ are non decreasing. As $\xi \rightarrow \pm \infty$ the trajectories of these solutions in the $(n+2+M)$-dimensional phase-space are tangent to the eigenvectors of the linearization matrices for system (5.1) at the starting and ending points of the heteroclinic solution respectively, i.e. at the points:

$$
\left(u, v_{1}, \ldots, v_{M}, z, z_{1}, \ldots, z_{M}, v_{M+1}, \ldots, v_{n}\right)=\left(u_{1}, v_{1}^{1}, \ldots, v_{M}^{1}, 0,0, \ldots, 0, v_{M+1}^{1}, \ldots, v_{n}^{1}\right)
$$


and

$$
\left(u, v_{1}, \ldots, v_{M}, z, z_{1}, \ldots, z_{M}, v_{M+1}, \ldots, v_{n}\right)=\left(u_{3}, v_{1}^{3}, \ldots, v_{M}^{3}, 0,0, \ldots, 0, v_{M+1}^{3}, \ldots, v_{n}^{3}\right)
$$

respectively. According to Lemma 5.2, the eigenvalues corresponding to these eigenvectors must be positive (as $\xi \rightarrow-\infty$ ) and negative (as $\xi \rightarrow \infty$ ). Moreover, the solutions are non decreasing, so if the components of these eigenvectors corresponding to $\left(u, v_{1}, \ldots, v_{n}\right)$ are chosen nonnegative, then the components corresponding to $\left(z, z_{1}, \ldots, z_{M}\right)$ should be nonnegative for $\xi=-\infty$ and non positive for $\xi=\infty$. The following additional properties can be proved.

Lemma 5.3. Let $q \neq 0, a \neq 0$ and Let $N=\left(h_{u}, h_{* 1}, \ldots, h_{* M}, h_{z}, h_{1}, \ldots, h_{n}\right)^{T}$ be an eigenvector of the linearization matrix (5.3) corresponding to an eigenvalue $\lambda$ such that:

1. if $\lambda>0$, then $N$ has nonnegative components,

2. if $\lambda<0$, then $h_{u} \geq 0, h_{* k} \geq 0, h_{z} \leq 0, h_{k} \leq 0$ for $k=1, \ldots, M$ and $h_{k} \geq 0$ for $k=M+1, \ldots, n$.

Then the inequalities concerning $\lambda$ and the components of the vector $N$ are strict.

Using Lemma 5.2 we can easily verify the validity of the next lemma.

Lemma 5.4. Let $L_{1}$ and $L_{3}$ denote the linearization matrices at the points $P_{1}$ and $P_{3}$ respectively. Then all the eigenvalues of both $L_{1}$ and $L_{3}$ have non zero real parts. The number of eigenvalues with positive and negative real parts is the same for $L_{1}$ and $L_{3}$.

Proof The first part follows from Lemma 5.2. Let $a^{[1]}, a_{k}^{[1]}, b_{k}^{[1]}$ and $a^{[3]}, a_{k}^{[3]}, b_{k}^{[3]}$ denote the parameters of the matrices $L_{1}$ and $L_{3}$ respectively. Let us define the homotopy $a(s)=$ $a^{[1]}+s\left(a^{[3]}-a^{[1]}\right), a_{k}(s)=a_{k}^{[1]}+s\left(a_{k}^{[3]}-a_{k}^{[1]}\right), b_{s}=b_{k}^{[1]}+s\left(b_{k}^{[3]}-b_{k}^{[1]}\right)$, with $s \in[0,1]$. During the homotopy the eigenvalues of the matrix determined by parameters corresponding to $s \in[0,1]$ change continuously. However their real parts cannot be equal to zero according to Lemma 5.2. The proof is complete.

Below, we will also need the following lemma.

Lemma 5.5. Consider system (1.9)-(1.10) (with $\left.D>0, D_{i}>0, i=1, \ldots, n\right)$. If one of the functions $z=u^{\prime}, z_{i}=v_{i}^{\prime}, i \in\{1, \ldots, n\}$, attains a minimum equal to zero for some $\xi=\xi_{0}$ then $z(\cdot), z_{1}(\cdot), \ldots, z_{n}(\cdot) \equiv 0$. The same is true for system (1.11)-(1.13).

Proof. The proof is straightforward.

Let, for $\alpha, \beta=0,1,2,3$, with $\Omega$ being a compact interval in $\mathbb{R}^{1}$ or $\Omega=\mathbb{R}^{1}$,

$$
B_{\alpha \beta}(\Omega)=S_{\alpha} \times \underbrace{S_{\alpha} \times \ldots \times S_{\alpha}}_{M \text { times }} \times \underbrace{S_{\beta} \times \ldots \times S_{\beta}}_{n-M \text { times }}
$$


where, for $\gamma=1,2,3$,

$$
S_{\gamma}=\left\{f \in C^{\gamma}(\Omega): \lim _{\xi \rightarrow \pm \infty} S_{\gamma}(\xi) \text { exist, } \lim _{\xi \rightarrow \pm \infty} S_{\gamma}^{(j)}(\xi)=0,1 \leq j \leq \gamma\right\}
$$

whereas

$$
S_{0}=\left\{f \in C^{0}(\Omega): \lim _{\xi \rightarrow \pm \infty} S_{0}(\xi) \text { exist }\right\} .
$$

Here, by $S_{\gamma}^{(j)}$ we mean the $j$-th derivative of $S_{\gamma}$. $B_{\alpha \beta}$ are the Banach spaces with the supremum norm. To be more precise

$$
\left\|\left(u, v_{1}, \ldots, v_{n}\right)\right\|_{B_{\alpha \beta}}=\|u\|_{S_{\alpha}}+\sum_{k=1}^{M}\left\|v_{k}\right\|_{S_{\alpha}}+\sum_{k=M+1}^{n}\left\|v_{k}\right\|_{S_{\beta}}, \quad\|\cdot\|_{S_{\gamma}}=\|\cdot\|_{C^{\gamma}(\Omega)} .
$$

Without losing generality we impose an additional condition on the heteroclinic solutions $U=\left(U_{1}, U_{2}, \ldots, U_{n+1}\right)$ (see $\left.(4.5)\right)$ to get rid of the translational symmetry. For instance we may choose it in the form

$$
U_{1}(0)=\left(u_{1}+u_{2}\right) / 2 \text {, }
$$

where $u_{1}, u_{2}$ are defined in Assumption 1.1. Let $D_{i}=\varepsilon d_{i+1}$, for $i=M+1, \ldots, n$ and $\varepsilon=1 / l$ with $l \in\{1,2, \ldots\}$. Thus we have a sequence of solutions $\left\{U_{[l]}\right\}_{l=1}^{l=\infty}$ and the corresponding speeds $\left\{q_{l}\right\}_{l=1}^{l=\infty}$.

By means of Lemma 4.1, $\left(q_{l}, U_{[l]}\right) \in \mathbb{R}^{1} \times B_{32}$ with $\left(\left\|U_{[l]}\right\|_{B_{32}}+\left|q_{l}\right|\right)$ bounded uniformly with respect to $l$. Using the Arzela-Ascoli lemma we conclude that on every compact interval $I_{K}=[-K, K], K>0$, we may find a subsequence $\left\{k_{l}\right\}_{l=1}^{l=\infty}$ such that both $U_{\left[k_{l}\right]}$ and $q_{k_{l}}$ are converging in $\mathbb{R}^{1} \times B_{21}\left(I_{K}\right)$ and $\mathbb{R}^{1}$ respectively. Next, from this subsequence we can choose another subsequence (denoted for simplicity in the same way) such that the sequences $U_{\left[k_{n}\right]}$ and $q_{k_{n}}$ are convergent in the norm of the space $\mathbb{R}^{1} \times B_{21}\left(I_{K+1}\right)$. This procedure can be continued. It follows that on every compact subset of the form $I_{N}=[-N, N]$ with natural $N$ arbitrarily large, we can find subsequences $\left\{U_{\left[k_{l}\right]}\right\}_{l=1}^{l=\infty}$ and $\left\{q_{k_{l}}\right\}_{l=1}^{l=\infty}$ converging to the solutions of the system (1.11)-(1.13). By differentiation of the equations of this system we easily conclude that the limiting pair $(Q, \Psi)$ belongs to the space $\mathbb{R}^{1} \times B_{32}$. One can prove that the function $\Psi(\cdot)$ connects the constant states $P_{1}$ and $P_{3}$ as was desired. So, according to the fact that the first derivatives of the functions $U_{[l]}, l=\{1,2, \ldots\}$, are positive in $\mathbb{R}^{1}$, and tend to zero at infinities, $\Psi^{\prime}(\xi)$ must tend to 0 for $|\xi| \rightarrow \infty$. Moreover, as $\xi \rightarrow \pm \infty$, due to the monotonicity, the functions $\Psi_{1}(\cdot), \Psi_{2}(\cdot), \ldots, \Psi_{n+1}(\cdot)$ must attain their limits. Due to condition (5.5), $\lim _{\xi \rightarrow-\infty} \Psi(\xi)=P_{1}$. Now, if it was not true that $\lim _{\xi \rightarrow \infty} \Psi(\xi)=P_{3}$, we would have $\Psi(\xi) \rightarrow P_{2}$ as $\xi \rightarrow \infty$. But it is easy to note that then we would have another wave $\widetilde{\Psi}$ such that $\widetilde{\Psi}(\xi) \rightarrow P_{2}$ and $P_{3}$ as $\xi \rightarrow \pm \infty$ respectively, that is to say there would exist two waves (of the same speed $q$ ) joining the states $P_{1}$ with $P_{2}$ and $P_{2}$ with 
$P_{3}$ consecutively. (Let us note that every $U_{[l]}(\cdot)$ is a $C^{1}\left(\mathbb{R}^{1}\right)$ function of its argument and $\lim _{\xi \rightarrow \infty} U_{[l]}(\xi)=P_{3}$. For the detailed considerations the reader is referred to [5] p.478.) If $Q \neq 0$ then this possibility should be however excluded due to Lemma 5.1. If $Q=0$, the first $M+1$ equations decouple from the rest. It is easy to note that for this system an analogous (to Lemma 5.1) property holds (see also [11]). Thus $\lim _{\xi \rightarrow \infty} \Psi_{1}(\xi)=P_{1}$ and consequently $\lim _{\xi \rightarrow \infty} \Psi(\xi)=P_{3}$ also in this case.

Let us remind that $q_{r}$ denotes the speed of the unique (in the sense of profile) monotonically increasing heterolinic solution for system (1.14)-(1.15) joining the states $\mathcal{P}_{1}$ and $\mathcal{P}_{3}$. The first existence result of the paper is contained in the following theorem.

Theorem 5.1. Suppose that Assumption 1.1 is satisfied. Then there exists a heteroclinic solution to system (1.11)-(1.13), i.e. a speed $q$ and a vector function $\left(u, v_{1}, \ldots, v_{n}\right): \mathbb{R}^{1} \rightarrow$ $\mathbb{R}^{n+1}$ satisfying conditions (1.8). This solution pair is a limit of the unique (up to a translation in $\xi$ ) heteroclinic pair for systems (1.9)-(1.10) (with $D_{i}>0$ ) as $D_{i} \rightarrow 0$ for $i=M+1, \ldots, n$. If $q_{r} \neq 0$ then also $q \neq 0$.

Proof. From the results obtained above it follows that we only need to prove the last statement concerning the relations between $q$ and $q_{r}$. This follows from Lemma 5.6, which is formulated and proved below.

Lemma 5.6. Assume that $q_{r} \neq 0$. Then for $\varepsilon \geq 0$ sufficiently small the heteroclinic pairs $(q, u)$ to system (1.11)-(1.13) described in Theorem 5.1 must satisfy the condition $q \neq 0$.

Proof. Suppose that there exists a subsequence $\left\{k_{l}\right\}_{l=1}^{l=\infty}$ such that $\left\{q_{k_{l}}\right\}_{l=1}^{l=\infty} \rightarrow 0$ as $l \rightarrow \infty$. Thus, according to Lemma 4.1 for every $\rho>0$ arbitrarily small there exists $l_{\rho}$ such that $\left|q_{k_{l}} v_{k_{l} i}^{\prime}(\xi)+1 / k_{l} D_{i} v_{k_{l} i}^{\prime \prime}(\xi)\right|<\rho, i=M+1, \ldots, n$, for all $l>l_{\rho}\left(\right.$ and all $\left.\xi \in \mathbb{R}^{1}\right)$. Using these estimates in the first $M+1$ equations of the system we obtain system (1.14)-(1.15) perturbed by terms of the order $O(\rho)$. For $\rho=0$ (or $l=\infty)$ we obtain exactly system (1.14)(1.15) (since from the last $n-M$ equations it would follow that $G_{i}\left(u(\xi), v_{i}(\xi)\right) \equiv 0, i=$ $M+1, \ldots, n)$. According to Theorem 2.1 in [11] this system has a unique monotonically increasing heteroclinic pair $\left(q_{r}, u_{r}\right)$ (with $q_{r} \neq 0$ by the assumption of the lemma). Hence we arrive at a contradiction with the fact that $q_{k_{l}} \rightarrow 0$.

Let us note that for $q_{r}=0$ proof of the existence of a solution is straightforward.

Lemma 5.7. Suppose that $q_{r}=0$. Then there exists a unique heteroclinic pair $(0, U)$ of system (1.11)-(1.13). This pair is unique (up to a translation in $\xi$ ) in the class of solutions with $q=0$ and monotone $U$.

Proof. Take $q=0$. Then, the first $M+1$ equations separate from the rest. According to our assumptions and from Theorem 2.1, p.15 in [11] (Theorem 1) it follows that there exists a unique monotonically increasing heteroclinic solution joining the corresponding constant states $\mathcal{P}_{1}$ and $\mathcal{P}_{3}$. The remaining $n-M$ equations have the form

$$
G_{i}\left(u(\xi), v_{i}(\xi)\right)=0, \quad i=M+1, \ldots, n
$$


Given $u(\xi)$, according to the form of the functions $G_{i}$, equations can be solved explicitly with respect to $v_{i}(\xi)$.

\section{Uniqueness of heteroclinic solutions}

In this section we will prove the uniqueness of the monotonically increasing heteroclinic pairs of system (1.11)-(1.13).

Suppose that there exist two heteroclinic solution pairs $\left(q_{1}, W_{1}\right)$ and $\left(q_{2}, W_{2}\right)$ to system (1.11)-(1.13) satisfying conditions (1.8). We know from Lemma 5.5 that both $W_{1}$ and $W_{2}$ are strictly monotone. Let us note that the difference $z=W_{2}-W_{1}$ satisfies the system of equations:

$$
\mathcal{D}_{i} z_{i}^{\prime \prime}(\xi)+q_{1} z_{i}^{\prime}(\xi)+\sum_{j=1}^{n+1} F_{i, j}\left[W_{1}(\xi)+\theta_{i}(\xi)\left(W_{2}(\xi)-W_{1}(\xi)\right)\right] z_{j}(\xi)=-\left(q_{2}-q_{1}\right) W_{2 i}^{\prime}(\xi)
$$

where, for all $\xi \in \mathbb{R}^{1}, 0 \leq \theta_{i}(\xi) \leq 1$ and $\mathcal{D}_{i}=0$ for $i=M+2, \ldots, n+1$. Here for simplicity we denoted

$$
\mathcal{D}_{1}=D, \quad \mathcal{D}_{i+1}:=D_{i}, \quad i=1, \ldots, M
$$

Both $W_{1}$ and $W_{2}$ tend to $P_{1}\left(P_{3}\right)$ as $\xi$ tend to $-\infty(\infty)$. According to Lemma 5.3 they attain their limits exponentially, so the matrices $F_{i, j}\left[W_{1}(\xi)+\theta_{i}(\xi)\left(W_{2}(\xi)-W_{1}(\xi)\right)\right], i, j=$ $1, \ldots, n+1$, also tend to their asymptotic limits exponentially. To proceed we will need the following lemma.

Lemma 6.1. Let the function $z: \mathbb{R}^{1} \rightarrow \mathbb{R}^{n+1}$ satisfy the system

$$
L z \leq-\delta \phi(\xi)
$$

where $\phi(\xi)>0, \xi \in \mathbb{R}^{1}, \delta \geq 0$, and, for $i=1, \ldots, n+1$,

$$
(L z)_{i}(\xi)=\mathcal{D}_{i} z_{i}^{\prime \prime}(\xi)+q z_{i}^{\prime}(\xi)+\sum_{j=1}^{n+1} B_{i j}(\xi) z_{j}(\xi)
$$

with $\mathcal{D}_{i}=$ const $\geq 0$. Suppose that $B(\xi)$ is $(n+1) \times(n+1)$ matrix function with nonnegative off-diagonal elements, continuous in $\xi$ and such that there exist its limits at infinities. Suppose that $B_{ \pm}=\lim _{\xi \rightarrow \pm \infty} B(\xi)$ are such that $B_{ \pm}$are irreducible and have their principal eigenvalue negative. Suppose that $\lim _{\xi \rightarrow \pm \infty} z(\xi)=0$. Then there exist numbers $r_{+}$and $r_{-}<r_{+}$such that if $z\left(r_{ \pm}\right)>0$ (component wise) then $z(\xi)>0$ for all $\xi>r_{+}$and $\xi<r_{-}$. 
Proof. By a natural modification of the proof of Lemma 5.3 p.213 in [11] to the systems which may contain equations of the first order, one concludes that $z(\xi) \geq 0$ for all $\xi \in$ $\mathbb{R}^{1} \backslash\left[r_{-}, r_{+}\right]$, where $r_{ \pm}$are such that $z\left(r_{ \pm}\right)>0$ and the principal eigenvalues of $B(\xi)$ are negative and $B(\xi)$ is irreducible for $\xi>r_{+}$and $\xi<r_{-}$. Using this property, we will prove that $z(\xi)>0$ for all such $\xi$. Let us consider the case $\xi>r_{+}$. Suppose that for some $\xi_{0} \in\left(r_{+}, \infty\right)$ the inequality $z\left(\xi_{0}\right)>0$ is not true. Let $I\left(\xi_{0}\right)=\left\{j: z_{j}\left(\xi_{0}\right)=0\right\}$ and $J\left(\xi_{0}\right)=\left\{j: z_{j}\left(\xi_{0}\right)>0\right\}$. Obviously $I\left(\xi_{0}\right) \cup J\left(\xi_{0}\right)=\{1, \ldots, n+1\}$. Suppose that $J=\emptyset$. Hence $z_{i}\left(\xi_{0}\right)=0$ for all $i$. If $\delta>0$, then the left hand side of the equation for $z_{i}$ is nonnegative whereas the right hand side is negative - a contradiction. If $\delta=0$, then from the uniqueness of solutions of the initial value problem, it follows that $z(\cdot) \equiv 0$ contradicting the assumption $z\left(r_{+}\right)>0$. So, there must exist at least one $j \in J\left(\xi_{0}\right)$ and at least one $i \in I\left(\xi_{0}\right)$ such that $B_{i j}\left(\xi_{0}\right)>0$ as otherwise the matrix $B$ would be reducible contrary to our assumptions. Thus we must have $z_{i}^{\prime}\left(\xi_{0}\right)=0$ and $z_{i}^{\prime \prime}\left(\xi_{0}\right) \geq 0$, if $\mathcal{D}_{i}>0$. But then Eq.(6.2) could not be satisfied since $z_{j}\left(\xi_{0}\right)>0$. The same considerations can be carried out for $\xi<r_{-}$. The lemma is proved.

Now, we are in a position to prove the following uniqueness result.

Theorem 6.1. Any two strictly monotone solutions $W_{1}$ and $W_{2}$ of system (1.11)-(1.13) corresponding to the speeds $q_{1}$ and $q_{2}$ respectively and satisfying conditions (1.8) coincide up to a shift and $q_{1}=q_{2}$.

Proof. Without losing generality we can suppose that $q_{2} \geq q_{1}$. Let us remind that, according to Lemma 2.8, $\partial F\left(W_{1}(\xi)\right)$ and $\partial F\left(W_{2}(\xi)\right)$ are irreducible for all $\xi$. Their principal eigenvalues are negative at $\pm \infty$. For fixed $W_{1}$ and $W_{2}$ let $r>0$ be such that $W_{i}(\xi)$ belong to sufficiently small neighborhoods of the points $P_{1}$ and $P_{3}$ if $|\xi|>r$. Then the principal eigenvalues of both $\partial F\left(W_{1}(\xi)\right)$ and $\partial F\left(W_{2}(\xi)\right)$ are negative for $|\xi| \geq r$.

Let us consider the function $W_{2 h}(\xi):=W_{2}(\xi+h)$. It is obvious that taking sufficiently large $h>0$ we can achieve that $W_{2}(r+h)>W_{1}(r)$. Moreover, for $\xi=-r-h$ we have

$$
W_{2 h}(-r-h)=W_{2}(-r-h+h)=W_{2}(-r)>W_{1}(-r-h),
$$

for $h$ sufficiently large. So, increasing the value of $h$ if necessary, we can guarantee that, taking $r_{+}=r$ and $r_{-}=-r-h$, we have $z(\xi, h):=W_{2 h}(\xi)-W_{1}(\xi)>0$ for all $\xi \in\left[r_{-}, r_{+}\right]$.

Since the principal eigenvalue of the matrix $\partial F$ is negative in some neighborhoods of the points $P_{1}$ and $P_{3}$ and due to the choice of $r$, the principal eigenvalue of the matrix with the elements $F_{i, j}\left[\left(1-\theta_{i}(\xi)\right) W_{1}(\xi)+\theta_{i}(\xi) W_{2 h}(\xi)\right], i, j=1, \ldots, n+1$ is also negative for $\xi>r$ and $\xi<-r-h$ and for all $h \geq 0$.

Thus, we may take $r_{-}=-r-h$ and $r_{+}=r$ in Lemma 6.1. Consequently, $z(\xi, h)>0$ for all $\xi \in \mathbb{R}^{1}$. Now, let us decrease $h$ and find its first value, $h=h_{0}$, for which the above condition does not hold. Let us note that if $\omega_{0}=\left\{\xi_{0}: z_{i}\left(\xi_{0}, h_{0}\right)=0\right.$ for some $i=$ $1, \ldots, n+1\}$, then there exists at least one $x_{0} \in \omega_{0} \cap\left[r_{-}, r_{+}\right]$(according to Lemma 6.1 and the conditions imposed on the value of $r)$. Let $\xi_{0}$ be such that the inequality $z\left(\xi_{0}, h_{0}\right)>0$ is not true. Let $I\left(\xi_{0}, h_{0}\right)=\left\{i: z_{i}\left(\xi_{0}, h_{0}\right)=0\right\}$ and $J\left(\xi_{0}, h_{0}\right)=\left\{j: z_{j}\left(\xi_{0}, h_{0}\right)>0\right\}$. Obviously $I\left(\xi_{0}, h_{0}\right) \cup J\left(\xi_{0}, h_{0}\right)=\{1, \ldots, n+1\}$. Suppose that $J\left(\xi_{0}, h_{0}\right)=\emptyset$. Hence $z_{i}\left(\xi_{0}, h_{0}\right)=0$ for all $i$ and for all $i=1, \ldots, n+1$ the equation for $z_{i}\left(\xi, h_{0}\right)$ at point $\xi=\xi_{0}$ would have the form 


$$
\mathcal{D}_{i} z_{i}^{\prime \prime}\left(\xi_{0}, h_{0}\right)=-\left(q_{2}-q_{1}\right) W_{2 h_{0} i}^{\prime}\left(\xi_{0}\right),
$$

due to the fact that $F_{i}\left(W_{2 h_{0}}\left(\xi_{0}\right)\right)-F_{i}\left(W_{1}\left(\xi_{0}\right)\right)=0$. As $\mathcal{D}_{i} z_{i}^{\prime \prime}\left(\xi_{0}\right) \geq 0$ we arrive at contradiction, unless $q_{2}=q_{1}$. If the latter condition is satisfied, then from the uniqueness of solutions of the initial value problem it follows that $z(\xi) \equiv 0$. Suppose that there exists at least one $i \in I\left(\xi_{0}, h_{0}\right)$ and at least one $j \in J\left(\xi_{0}, h_{0}\right)$ such that $F_{i, j}(W)>0$ for $W \in\left[P_{1}, P_{3}\right]$. Such a pair must exist as otherwise the matrix $B$ would be reducible contrary to our assumptions. But then the equation for $z_{i}\left(\xi, h_{0}\right)$ :

$$
\mathcal{D}_{i} z_{i}^{\prime \prime}\left(\xi_{0}, h_{0}\right)+q_{1} z_{i}^{\prime}\left(\xi, h_{0}\right)+F_{i}\left(W_{2 h_{0}}\left(\xi_{0}\right)\right)-F_{i}\left(W_{1}\left(\xi_{0}\right)\right)=-\left(q_{2}-q_{1}\right) W_{2 h_{0} i}^{\prime}\left(\xi_{0}\right)
$$

which at point $\xi=\xi_{0}$ reads

$$
\mathcal{D}_{i} z_{i}^{\prime \prime}\left(\xi_{0}, h_{0}\right)+F_{i}\left(W_{2 h_{0}}\left(\xi_{0}\right)\right)-F_{i}\left(W_{1}\left(\xi_{0}\right)\right)=-\left(q_{2}-q_{1}\right) W_{2 h_{0} i}^{\prime}\left(\xi_{0}\right)
$$

cannot be satisfied since $z_{i}^{\prime \prime}\left(\xi_{0}, h_{0}\right) \geq 0, F_{i}\left(W_{2 h_{0}}\left(\xi_{0}\right)\right)-F_{i}\left(W_{1}\left(\xi_{0}\right)\right)>0$ (due to the fact that $\left.z_{j}\left(\xi_{0}\right)>0\right)$ and by assumption $\left(q_{2}-q_{1}\right) \geq 0$ and $W_{2 h_{0}}^{\prime}(\xi)>0$. As $h_{0}$ with the above properties must exist, then we conclude that $W_{2 h_{0}}(\xi)=W_{1}(\xi)$ for all $\xi \in \mathbb{R}^{1}$ and $\left(q_{2}-q_{1}\right)=0$. The lemma is proved.

Finally, let us consider the perturbed system:

$$
\begin{gathered}
\mathcal{D}_{i} U_{i}^{\prime \prime}+q U_{i}^{\prime}+F_{i}(U)+\Phi_{i}(\tau, q, U)=0, \quad i=1, \ldots, M+1, \\
q U_{i}^{\prime}+F_{i}(U)+\Phi_{i}(\tau, q, U)=0, \quad i=M+2, \ldots, n+1,
\end{gathered}
$$

where $\tau \in \mathbb{R}^{l}$ is sufficiently close to 0 . Let us note that in fact $\Phi_{i}$ may depend on $\xi$. (See (6.1) for the definition of $\mathcal{D}_{i}$.)

Assumption 6.1. Suppose that, for $i=1, \ldots, n+1, \Phi_{i}: \mathbb{R}^{l} \times \mathbb{R}^{1} \times \widetilde{B}_{21} \rightarrow B_{00}, l \geq 1$ and $\Phi_{i}(0, q, U) \equiv 0$. Assume that for $\tau=0$ there exists a unique (up to translation in $\xi$ ) heteroclinic pair $\left(q_{0}, U_{0}\right)$ for system (6.4) joining the states $P_{1}$ and $P_{3}$ with $U_{0}(\cdot)$ monotonically increasing. Suppose that $\Phi$ is continuously Fréchet differentiable with respect to $(q, U)$ in some open neighbourhood of the solution triple $(\tau, q, U)=\left(0, q_{0}, U_{0}\right)$.

We are now able to formulate the result on the structural stability of the heteroclinic solution pairs for system (1.11)-(1.13).

Theorem 6.2. Suppose that $q_{r} \neq 0$ and that Assumptions 1.1 and 6.1 are fulfilled. Then for all $0 \leq|\tau|<\tau_{0}$, with $\tau_{0}$ sufficiently small, there exists a unique heteroclinic pair $\left(q_{\tau}, U_{\tau}\right) \in$ $\mathbb{R}^{1} \times \widetilde{B}_{21}$ for system (6.4) such that

$$
\left|q_{\tau}-q_{0}\right|+\left\|U_{\tau}-U_{0}\right\|_{B_{21}} \rightarrow 0
$$

as $|\tau| \rightarrow 0$.

The proof uses the properties of the linearized operator and is left to the reader. 


\section{Acknowledgements}

The authors express their gratitude to Elaine Crooks for helpful remarks. This paper was partially supported by the Polish Ministry of Science and Higher Education Grant No. 1P03A01230.

\section{References}

[1] E. C. M. Crooks, J. F. Toland, Travelling waves for reaction-diffusion-convection systems, Top. Meth. in Nonl. Anal., 1998, Vol.11, 19-43.

[2] M. Crandall, An Introduction to constructive aspects of bifurcation theory and implicit function theorem, in: P. Rabinowitz (Ed.), Applications of Bifurcation Theory, 1977, Academic Press, New York.

[3] M. Falcke, Reading the patterns in living cells - the physics of $\mathrm{Ca}^{2+}$ signaling, Advances in Physics, 2004, Vol.53, 255-440.

[4] F. R. Gantmaher, Teoria Matric (in Russian), Nauka, 1988.

[5] B. Kazmierczak, V. Volpert, Existence of heteroclinic orbits for systems satisfying monotonicity conditions, Nonlinear Analysis, 2003, Vol.55, 467-491

[6] K.J.Palmer, Exponential dichotomies and transversal homoclinic points, J. Diff. Equat., 1984, Vol.20, 225-256.

[7] J. Shenq Guo, J. Tsai, The asymptotic behavior of solutions of the buffered bistable system, J. Math. Biol., 2006, Vol.53, 179213.

[8] J. Sneyd, P.D. Dale and A. Duffy, 1998, Traveling waves in buffered systems: applications to calcium waves, SIAM Journal on Applied Mathematics, 1998, Vol.58, 1178-1192.

[9] A.E. Taylor, Introduction to functional analysis, 1958, J.Wiley and Sons, New York.

[10] J. Tsai, J. Sneyd, Existence and stability of traveling waves in buffered systems, SIAM J. Appl. Math., 2005, Vol. 66, 237-265.

[11] A. Volpert, V. Volpert, V. Volpert, Travelling Wave Solutions of Parabolic Systems, 1994, AMS, Providence. 